\title{
Real-Time Smart Parking Systems Integration in Distributed ITS for Smart Cities
}

\author{
Muhammad Alam, ${ }^{1}$ Davide Moroni $\mathbb{D}^{2},{ }^{2}$ Gabriele Pieri $\mathbb{D}^{2},{ }^{2}$ Marco Tampucci, ${ }^{2}$ \\ Miguel Gomes, ${ }^{3}$ José Fonseca, ${ }^{1}$ Joaquim Ferreira $\mathbb{D}^{1},{ }^{1}$ and Giuseppe Riccardo Leone ${ }^{2}$ \\ ${ }^{1}$ Instituto de Telecomunicações, Universidade de Aveiro, Portugal \\ ${ }^{2}$ Institute of Information Science and Technologies, National Research Council of Italy, Pisa, Italy \\ ${ }^{3}$ Micro I/O, Sistemas Electrónicos, Lda, Aveiro, Portugal \\ Correspondence should be addressed to Gabriele Pieri; gabriele.pieri@isti.cnr.it
}

Received 18 June 2018; Revised 7 September 2018; Accepted 24 September 2018; Published 21 October 2018

Academic Editor: Eneko Osaba

Copyright (C) 2018 Muhammad Alam et al. This is an open access article distributed under the Creative Commons Attribution License, which permits unrestricted use, distribution, and reproduction in any medium, provided the original work is properly cited.

\begin{abstract}
Intelligent Transportation Systems (ITS) have evolved as a key research topic in recent years, revolutionizing the overall traffic and travel experience by providing a set of advanced services and applications. These data-driven services contribute to mitigate major problems arising from the ever growing need of transport in our daily lives. Despite the progress, there is still need for an enhanced and distributed solution that can exploit the data from the available systems and provide an appropriate and real-time reaction on transportation systems. Therefore, in this paper, we present a new architecture where the intelligence is distributed and the decisions are decentralized. The proposed architecture is scalable since the incremental addition of new peripheral subsystems is supported by the introduction of gateways which requires no reengineering of the communication infrastructure. The proposed architecture is deployed to tackle the problem of traffic management inefficiency in urban areas, where traffic load is substantially increased, by vehicles moving around unnecessarily, to find a free parking space. This can be significantly reduced through the availability and diffusion of local information regarding vacant parking slots to drivers in a given area. Two types of parking systems, magnetic and vision sensor based, have been introduced, deployed, and tested in different scenarios. The effectiveness of the proposed architecture, together with the proposed algorithms, is assessed in field trials.
\end{abstract}

\section{Introduction}

The advancements in urbanization and the dependence on transportation lead to the evident increase in the number of vehicles on the roads around the world, which have a negative impact on the quality of life. Congestion on the roads, $\mathrm{CO} 2$ emission, energy waste, road accidents, and increase stress level are some of the problems that are seriously affecting public health and wasting useful resources. It is clear that innovative solutions are needed, and that is where Intelligent Transportation Systems (ITS) should play the role in contributing to tangible results quickly and efficiently. Therefore, new disruptive technologies and techniques that ensure ITS to be more efficient, safer, and energetically sustainable need to be introduced.
The development of urban centers and the increasing number of vehicles on the roads around the world lead to the evident increase in demand for parking places in urban centers, which seriously affects the quality of the traffic. The search for the available parking place increases the traffic volume; it is estimated that more than $70 \%$ of the European population lives in urban centers under almost constant impacts of air pollution and noise caused by road traffic [1]. But, next to the pollution, the problem called cruising for parking causes more than $30 \%$ of the usual total traffic congestion resulting from the daily travels and traffic jams due to road constructions, traffic accidents, and related traffic problems. The average search for the available parking place is up to 20 minutes. According to other conducted studies this percentage could be much higher and so in a borough of 


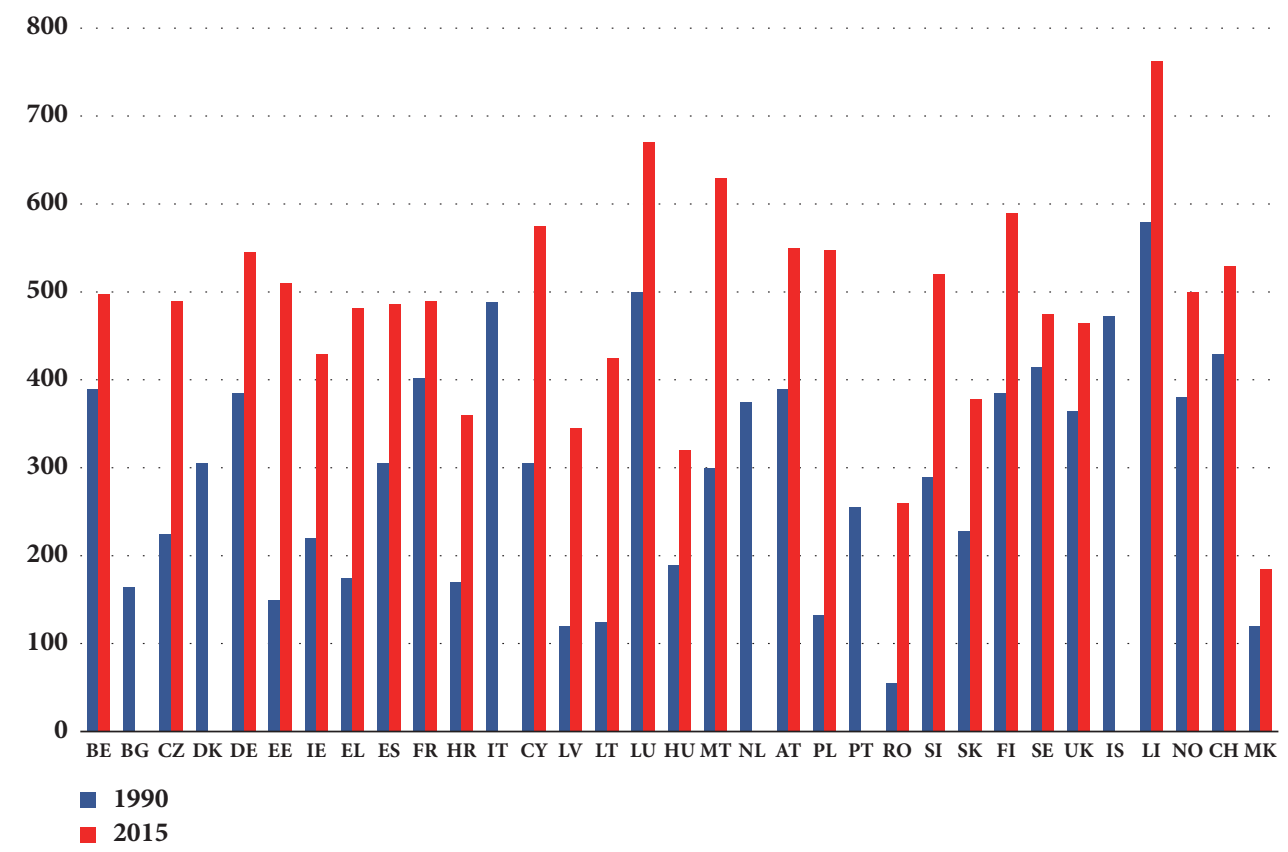

FIGURE 1: The increase in the number of passenger vehicles per 1000 inhabitants in EU [1].

Munich (Schwabing) even exceeds $44 \%$ and in a borough of Brooklyn, NY (Park Slope) exceeds 45\%. Moreover, according to an earlier study [1], 24\% of all traffic accidents are caused by careless parking (entry and exit from the parking lot), reckless departing from vehicles, sudden stops, etc.

In addition, from an economic point of view, in 2010, the specified congestion caused in America 4.8 billion hours of delay, 7.2 billion litres of additional fuel consumption, and the cost of 101 billion dollars. Only in Los Angeles the search for available parking places took 178,000 litres of fuel (which is enough to circle the world 38 times) and produced 730 tons of carbon dioxide [2].

The importance of applying the adequate strategy for the management of parking places can also be found in the estimate that during the day a typical vehicle stays in idle $95 \%$ of the time.

The driver's behavior, when choosing the type and the location of the parking place as well as making the final decision, depends on many factors and represents a considerably more complex process, which should be paid much more attention than before. The usage of advanced technologies is unavoidable in achieving selected combination of strategies.

Increased vehicle production in the last 30 years and higher purchasing power of citizens consequently induced extremely high number of vehicles which are daily or periodically using highways as shown in Figure 1. In addition, according to the report presented in [4], every car is parked for 23 hours (average) daily and thus makes it extremely difficult to find an empty parking. Today ITS are very complex systems, made of several subsystems working in isolation, which are often closed, i.e., they do not provide interfaces for direct access to third parties, and vertical, i.e., they only provide an end-to-end system from sensors/actuators to the human-machine interface (HMI) of the system. Hence, the fusion of data originated from subsystems operating in the same area is very difficult in practice and is bound to become worse in the future as the ICT infrastructure in transportation systems becomes bigger and more complex. Therefore, in this paper, we present an architecture which makes such interaction between heterogeneous data sources as seamless as possible, and which leverages such opportunity to shift the intelligence for decision making from humans in control centers to distributed machines. The proposed architecture tackles the problem of traffic management for the specific case of open parking space management, through local information diffusion on vacant parking slots to drivers. To this end, two types of parking systems' technologies are considered: using magnetic and vision sensor based. They were considered, deployed, and tested in different urban scenarios, and the effectiveness of the proposed architecture, along with the proposed algorithms, was assessed in field trials.

This paper is further organized as follows: the related work is presented in Section 2, Section 3 presents the proposed distributed architecture, and Section 4 presents vision based algorithm. The implementation of both magnetic and vision sensors are presented in Section 5 while Section 6 describes the equipments installation. The conclusions are presented in Section 7.

\section{Related Work}

As urban centers are becoming increasingly more populated and congested, the management of public parking places, according to local mobility policies, is a key strategic issue with high economical impact. Academia and industry are 
well aware of the potential gains that can be obtained taking advantage of information and communication technologies applied to this field. An efficient smart parking system will favor drivers, by reducing the time to find a parking place and by optimizing parking spaces' utilization rate, thus increasing the revenues for the urban parking operators.

The demand for the available parking place is a dynamic value, i.e., a value that changes over time, so logically there is a need to provide the information on the location and condition of parking offer in real time to the drivers of passenger cars. The systems for providing the information on parking offer to users already began to be used in some world cities more than 20 years ago and can be found under names like "A Guided Parking System" or "Dynamic Parking Guidance System" [5]. By the usage of Parking Guidance and Information (PGI) systems it is possible to improve to some extent the process of parking and increase its success and efficiency. The ecological effects of potential PGI systems application are evident in the reduction of noise (the decreasing number of vehicles in certain segments of the transport network) and in the reduction of fuel consumption and exhaust emissions (shorter travel times and the increase in the traffic flow).

Typically, a PGI consists of 4 components, namely, the parking lot monitoring, information dissemination, communication technology, and the control system [6]. The PGI systems use either smart sensors or cameras for monitoring lots occupancy. The sensors based monitoring is further classified as "On-Roadway" or "Off-Roadway". The on-road sensors are glued to the road surface; examples of such type of sensors are magnetic sensors and acoustic sensors. The offroad sensors are placed above the surface of the road; the examples are smart cameras for monitoring.

The body of knowledge in this area is vast, with largescale field deployments since 1996. Lin et al. [7] provide an exhaustive, although not systematic, survey on smart parking solutions, from 2000 to 2016, and identify some challenges and open issues. The smart parking system can be designed in several ways and there are different technology options available. Regarding the technologies available for parking lots monitoring various sensor solutions such as magnetic sensor, acoustic sensors, RFIDs, ultrasounds, inductive loops, smart cameras, etc. can be used. Recently more advanced solutions for street parking detection and monitoring systems are proposed, for example, crowdsourcing [8], which is based on an ultrasonic range finder placed in each vehicle to detect free places near the parked vehicle. The collected information is then disseminated to the other vehicles' drivers to help them find these free slots.

In [9] the authors have used photoelectric sensors to detect the vehicles in a controlled zone. The sensors were installed at the entry/exit of the parking and connected with the server for information processing and dissemination. Such arrangement can provide real-time information about the number of free parking lots but does not provide exact location of the free space. In addition, the problem of finding exact free parking lot grows exponentially with the number of parking lots. An increased production of sensors and smart cameras has enhanced the scope of analysis and management of urban traffic activities. New disruptive techniques leveraging computer vision that provides an added value to cameras by automatically extracting relevant information have been proposed in several works. Therefore, these new techniques based on computer vision and video analytics become increasingly important for ITS. For instance, a smart camera based real-time car parking monitoring systems exploiting the convolutional neural network algorithms was presented in [10]. The system was tested and validated for robustness and effectiveness in various challenging scenarios such as low light, shadows, and occlusions. The presented system exhibits high performance; however it is not integrated with a fully distributed ITS architecture. In [11] the authors have used applied computational mechanisms and image processing techniques to monitor and authorize vehicles entering the parking area. However, the presented systems only detect and recognize the vehicles and verify if they are the registered users or not. Recently, a more interesting work on image processing for smart transport infrastructure is presented in [12] using drones. The presented work uses drone cameras to capture the videos and then analyze it for the available parking lots and congestion on the roads. However, the presented work shows less precision and also not fully integrated into a distributed ITS system. Other related works that use smart camera for parking lots monitoring can be found in [13-16].

The work presented in this paper addresses some of the challenges and openes issues identified in [7], notably at the sensing layer, guaranteeing personal privacy, and by using smart, low power, magnetic, and vision sensors. Moreover, with the aim of designing an architecture which can have high scalability and reliability, in the following section our fully distributed ITS is presented and described, along with the two different sensing technologies for covering different aspects of parking systems (e.g., outdoor and underground parking lots). Another issue, faced by our proposal, regards the conditions for proposing a low-cost solution, at least in comparison with other proposals mentioned above, and with regard to several aspects: from the sensors customization to the infrastructures and from the maintenance to the scalability.

\section{Distributed ITS Architecture}

In current information systems, supporting ITS applications, data flows from the edges of the system, i.e., sensors, road-side units, vehicles, and personal devices, towards a centralized data aggregation and control center. Automated or manual control and supervision decisions are then either taken and fed back to the edge actuation components or forwarded externally, e.g., to databases or web services. This architecture has a number of drawbacks, such as the single point of failure of the central subsystem (control center) and the increased end-to-end delay. Moreover, this legacy architecture has limited scalability and availability, as the addition of new road sections implies new configurations, parameterizations, and system restart. Therefore, based on these limitations, a different architecture was proposed, in the scope of the 


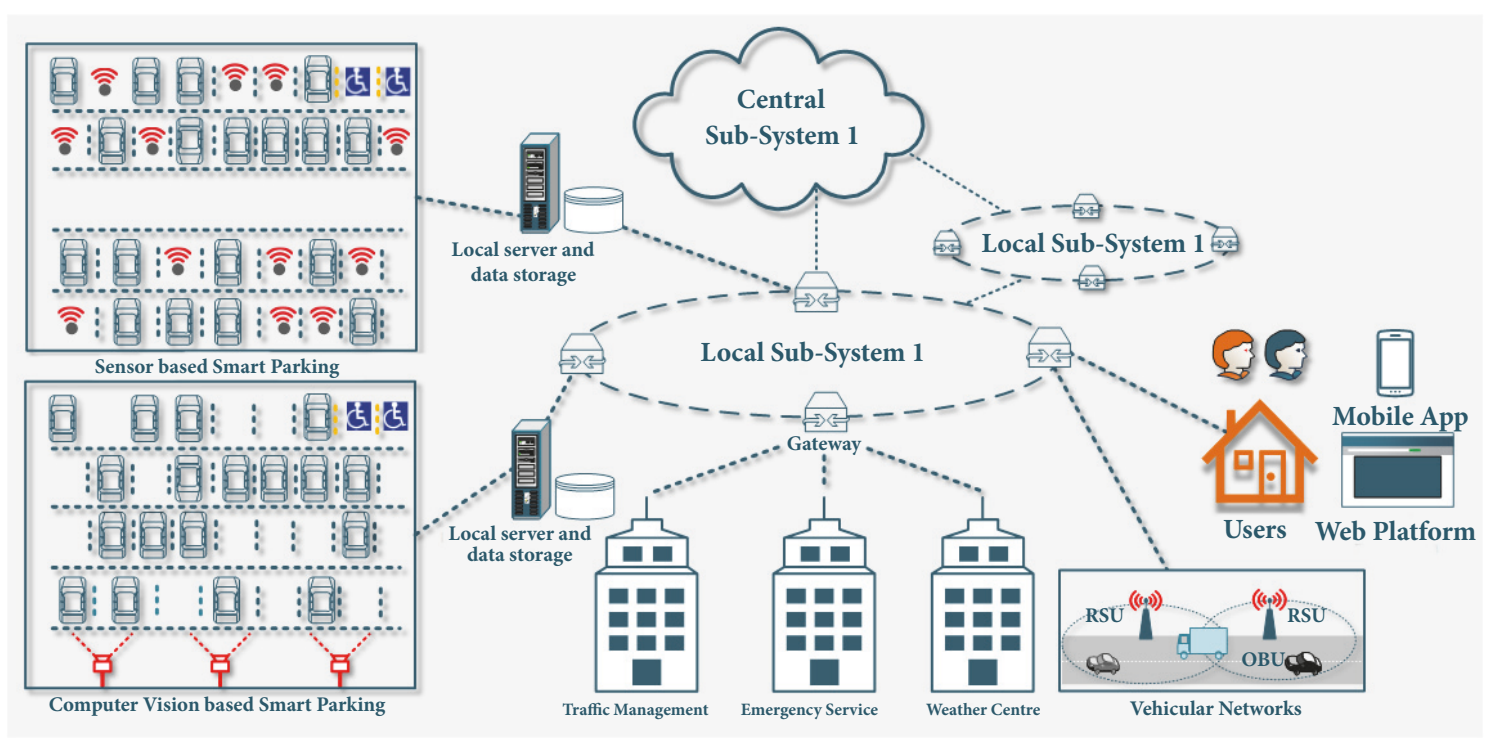

FIgURE 2: Distributed ITS architecture.

ICSI project [17], where the intelligence and storage are distributed across limited geographical areas, as depicted in Figure 2. In this architecture, data is processed in gateways located at each local subsystem without the intervention of the control center; hence the information is locally distributed. These gateways aggregate and store data, process events, issues warnings, etc., within a bounded geographical area. In turn, smart parking systems, vehicular networks via road-side units (RSUs), and other personal subsystems are connected with gateways. Additionally, each gateway is logically connected to the control center, for long-term data aggregation, optimizations, and big data processing. The proposed architecture provides higher dependability and reliability, as faults are confined within the respective gateway, while other gateways and the control center may continue to operate without failures. Furthermore, the new architecture is highly scalable, since the addition of new components of the system is automatically accommodated by a proportional increase of the number of gateways. Thus, thinking to the typical development in an urban environment, new clusters (e.g., an existing parking lot becoming monitored by smart cameras) could be added with only the cost of the infrastructure and the nodes, but at almost no cost for the whole architecture. In addition, reactions in bounded time are favored, because the complexity of interactions is limited to a local scope and the communication path is much shorter. A data distribution layer acts as a bridge between the cooperative sensing infrastructure (sensors, road-side units, vehicles, and personal devices), the local gateways, and the central subsystem. The data distribution layer is connected to the border routers of the vehicular network and the smart parking subsystems using the CoAP protocol. The border router communicates with On-Board Units (OBUs) using the ETSI ITS G5 and with the parking sensors and cameras using 6LoWPAN.

\section{Design of Cooperative Computer Vision Algorithms for Real-Time Parking Lot Monitoring}

For the analysis of parking lot occupancy status, the approach followed is based on an ad hoc computer vision pipeline capable of highlighting the changes in the Regions of Interest (RoI), with respect to an adaptive background reference image. The pipeline, described in the following, is particularly suitable to real-time deployment on resource-constrained embedded devices.

Change detection: in order to improve the computational efficiency, the frame differencing for detecting changes is performed only on predetermined RoI in the acquired frame. Each of the RoI corresponds to a specific parking slot, and for each of the regions the absolute accumulated pixelwise difference is reported; such a difference is dynamically weighted in order to correct and improve the robustness of the algorithm with respect to environmental light changes. In order to perform this improvement, normalized versions of the images are computed and used, with respect to global illumination parameters (average and variance of both the current and reference image). The sum of the differences is scaled with respect to the size of the RoI, and finally it is stored in a buffer. At this point verification occurs in order to detect the possible change. In particular, a comparison of the stored actual value with the historical values allows filtering out possible spurious values (i.e., exceeding the threshold) due to, e.g., the presence of shadows. In the same way the stored value is compared with another threshold in order to detect possible changes with respect to the reference image. At this point the algorithm yields a first outcome which is a value regarding the occupancy status of the specific parking slot. Intranode occupancy detection: once the algorithm has computed values regarding the occupancy for each parking slot (corresponding to the RoIs), an 
intranode occupancy detection process occurs. In order to avoid transitory events (e.g., people crossing by and shadows casted by external objects), the occupancy status becomes effective and is transmitted to the smart camera network, only after being observed consecutively for a specific number of acquired frames. For each parking slot, the algorithm yields a confidence value in the range [0..255], meaning that values next to 0 represent an almost no-change detected with respect to the reference value, and thus the slot is likely to be free; higher values, on the other hand, indicate that main changes have occurred in the observed scene, and thus the slot is likely to be occupied.

Internode cooperative decision making: the confidence values produced by the single nodes as a 256 levels number should be transformed to binary values corresponding either to free or busy parking slots, thus taking a final decision regarding the parking availability. To this end, local confidence values will be propagated through the smart camera network thanks to its middleware. In particular when a parking space is monitored by more than one sensor node, the final decision regarding its occupancy is obtained in a cooperative way, at an internode level. This final decision is obtained aggregating all the confidence values produced by the different nodes (which are statically dislocated and have static tables of the monitored parking slots). If a slot $k$ is monitored by $n=n(k)$ sensor nodes and $v_{1}^{k}(t), \ldots, v_{n}^{k}(t)$ are the confidence values measurements from each single sensor node at time $t$, then the aggregated measure is computed as

$$
v^{k}(t)=\sum_{i=1}^{n} \omega_{i, k} v_{i}^{k}(t)
$$

where $\omega_{i, k}$ are the nonnegative weights and

$$
\sum_{i=1}^{n} \omega_{i, k}=1
$$

Thus the final decision $s t^{k}(t)$ regarding the slot $k$ is obtained performing a comparison with a threshold $\varepsilon$ :

$$
s t^{k}(t)= \begin{cases}1 & \text { if } v^{k}(t)>\varepsilon \\ 0 & \text { if } v^{k}(t) \leqslant \varepsilon\end{cases}
$$

For the implementation of a more robust algorithm that avoids meaningless oscillations, the above decision is further improved using two levels of thresholds $\varepsilon_{1}<\varepsilon_{2}$ and considering the status of the slot at the previous measure obtained at time $t-1$ :

$$
\begin{aligned}
& s t^{k}(t) \\
& = \begin{cases}1 & \text { if } v^{k}(t) \geq \varepsilon_{2} \text { or }\left(v^{k}(t)>\varepsilon_{1} \text { and } s t^{k}(t-1)=1\right) \\
0 & \text { if } v^{k}(t) \leq \varepsilon_{1} \text { or }\left(v^{k}(t)<\varepsilon_{2} \text { and } s t^{k}(t-1)=0\right)\end{cases}
\end{aligned}
$$

Weights $\omega_{i, k}$ are determined heuristically for each physical configuration of the smart camera network, while the thresholds $\varepsilon_{1}, \varepsilon_{2}$ are set to a common value for all the nodes, the sensors, and the parking slots.

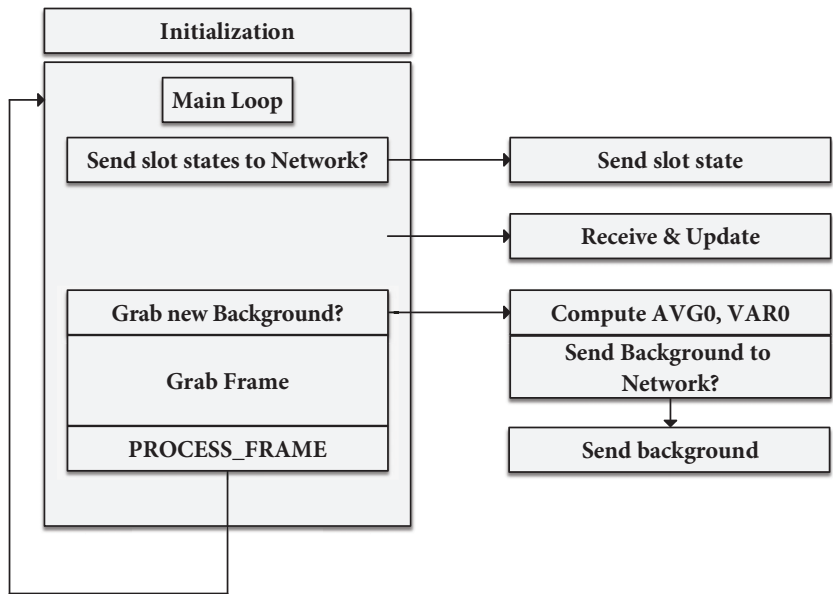

FIGURE 3: Parking lot occupancy flowchart in analysis software (this figure has been modified after [3] (Magrini et al., 2015)).

The general processing flow for the parking lot algorithm is presented in Figure 3. The main loop of the algorithm starts by possibly sending the status of the slot being monitored to the network. Afterwards, if needed, the new active background is acquired and stored, simultaneously with the computation of statistical data over the frame. Once the actual frame is acquired its real-time processing is performed. The processing follows the above described steps, from the normalization of the acquired frame to the final outcome evaluating the occupation status of the monitored slots. In the following cycle, the slot status are sent (if the communication is needed and foreseen to be performed at every single cycle) to the higher level of the network, where the computed values can be aggregated as previously described.

\section{Implementation}

\subsection{Sensor Based Smart Parking Implementation}

5.1.1. Architecture. Micro I/O has developed a smart parking system as depicted in Figure 4 that is suitable for both indoor and outdoor environments. The developed system is based on wireless magnetic sensors as depicted in Figure 5 that are deployed on the ground in each parking space for the respective occupancy detection. The system is compatible with multiple user platforms, for management and driver guidance to available spaces and interoperable with several complementary systems, such as LED panels, tags, indicators, alarms, and barriers. In terms of architecture, the system consists of three major layers:

(i) The endpoints layer, encompassing the parking sensors and actuators.

(ii) The communication layer, encompassing gateways and repeaters, to bridge the communications between the endpoints and the rest of the system.

(iii) The software layer, consisting on all cloud-based components responsible for data collection, parsing, 


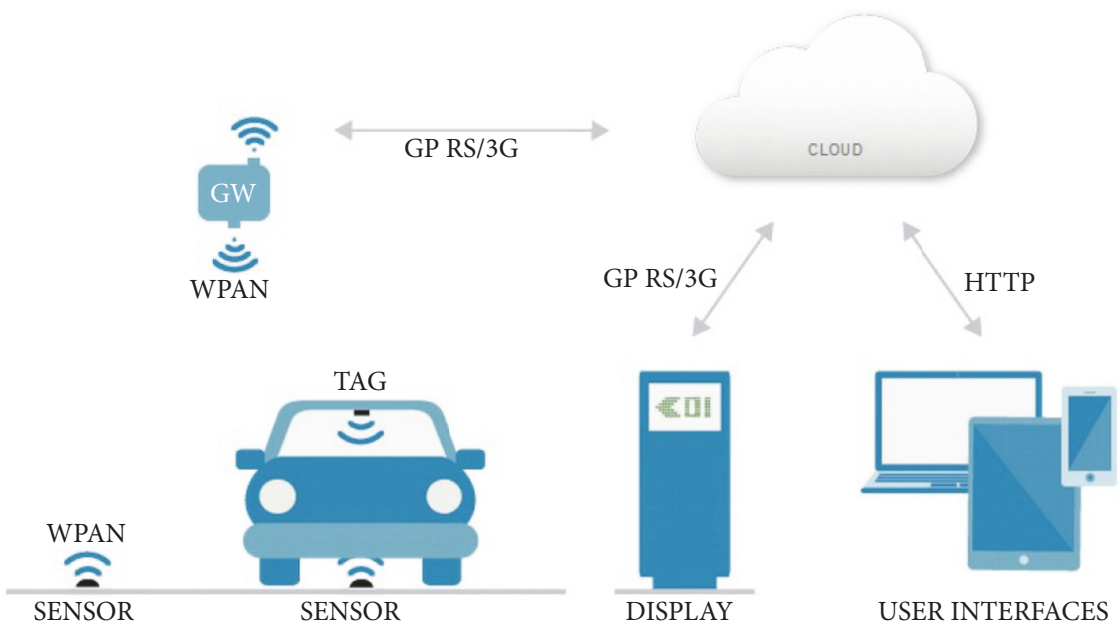

FIGURE 4: Sensor based smart parking overview.

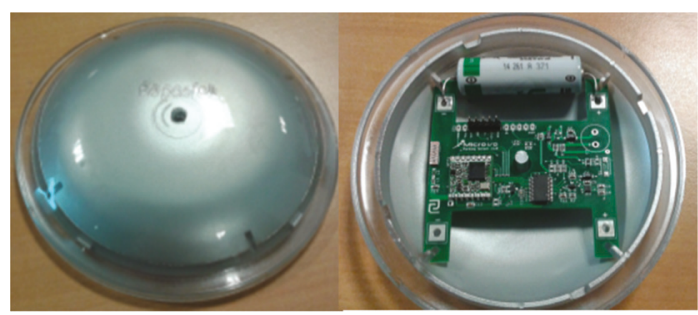

FIGURE 5: Deployed sensors.

storage, and provision, from the web services and databases, to the multiple user platforms.

The system has the following features and specifications:

(i) Encapsulation: the sensor does not require cabling, as it features wireless communications and internal batteries for power. The device is installed inside a plastic capsule, which is then filled with epoxy, and after the epoxy has hardened, the device is completely isolated. The encapsulation enables complete impermeability and the ability to withstand extreme pressure (as the weight of the vehicle).

(ii) Deployment: after encapsulated, the sensor is of easy and fast deployment. It only required the application of asphaltic glue beneath the sensor and little pressure after its deployment on the ground, followed by few waiting minutes for the glue to dry and attain a robust fixation to the ground.

(iii) Programming and firmware updates: the sensor firmware can be remotely transferred to the device. The system features FOTA (Firmware Over The Air).

(iv) Initialization: in order to initialize the sensor, a provided magnet has to be approximated to the sensor. The device includes a reed sensor that will detect the presence of the magnet and activate the device. (v) Detection: the sensor features a 3-axis digital magnetometer (Freescale MAG3110) for the vehicular detection, through the perception of the variations on the Earth's magnetic field, caused by the passage/presence of the vehicle above it. The sensor has a determined sampling rate and if the magnitude of the readings overcomes the algorithm's defined thresholds, the status change is communicated to the system.

(vi) Temperature compensation: the magnetometer sensibility varies with the temperature, being influenced both by the temperature of its surroundings and the exposure to sunlight. For proper operation, the sensor requires calibration and temperature compensation, in case of variations. For this purpose, the MAG3110 features a temperature sensor and samples are also taken along the magnetic readings, for compensation/recalibration, if necessary.

(vii) Communication: according to the application requirements, in terms of communications range and device autonomy, the chosen communications technology was LoRa, over $868 \mathrm{MHz}$. This technology enables a communications range up to $200 \mathrm{~m}$ and low power consumptions. There can be two types of communications:

(a) Status change notification: following a sampling event, the data is analyzed and, in case a status change is detected, the sensor sends the respective communication/notification to the system.

(b) I'm alive notification: if no status change is verified for a defined period of time, the sensor sends an "I'm alive" notification to the system, indicating it is operating properly.

(viii) Power management and autonomy: the device autonomy represents its lifetime. The sensors can feature up to 4 AA low self-discharge Li-SOCl2 batteries (SAFT LS14500, 3.6V, 2.6Ah); however, due to the permanent 
epoxy encapsulation, the batteries are not replaceable nor rechargeable; therefore power management is of great relevance. In order to expand the device's lifespan, the processes involving sampling, data processing, and communications were optimized:

(a) Sampling: in order to reduce the time the microprocessor is awake, but not compromise the quality of service levels, by ensuring prompt status updates, the defined sampling periodicity is of 4 seconds. After the device is activated, at every 4 seconds the microprocessor will wake up from sleep, take a sample and, if no change is detected, go back to sleep;

(b) Processing: in order to reduce power consumption, the selected microprocessor was the (Low Power) Microchip PIC18LF26K22-I/SS. Its power consumption in sleep mode (at which it is most of the time) is, approximately, $1 \mu \mathrm{A}$.

(c) Communications: communications are the most power consuming processes. The chosen communications technology, LoRa on the $868 \mathrm{MHz}$ band, enables low power consuming communications, but the periodicity and duration (size) of the communications was also optimized, for greater power savings.

According to the defined periodicity of the "I'm alive" messages, for example, with intervals not smaller than 30 minutes, the sensors can achieve an autonomy beyond 8 years.

5.2. Vision Based Smart Parking. In this section, a smart camera prototype for vision based smart parking is presented. The prototype is based on previous work $[3,18]$; choices that lead to the selection of specific hardware are motivated and discussed and technical facts are reported. Main emphasis is given to the vision board, which is a custom Printed Circuit Board (PCB) manufactured specifically for the sensor. Cameras, wireless module, batteries, photovoltaic panels, and housing are also briefly addressed.

5.2.1. Vision Board. For the implementation and development of the vision board, an embedded Linux architecture has been selected that provide enough computational power and can be easily programmed. A selection of ready-made Linux based prototyping boards had been evaluated with respect to computing power, flexibility, price, and support. The following candidates were considered: Raspberry Pi Model B (ARM11, $700 \mathrm{MHz}$ ), Phidget SBC (ARM9, 400 $\mathrm{MHz}$ ), and BeagleBone-TI Sitara AM3359 (Cortex A8, 720 $\mathrm{MHz}$ ). All the candidates have as common disadvantages high power consumption. It has been therefore decided to realize a custom vision component by designing, printing, and producing a new PCB. The new PCB has been designed to have the maximum flexibility of use while maximizing the performance/consumption ratio. A good compromise has been achieved by using a Freescale CPU based on the ARM architecture, with support for MMU-like operating

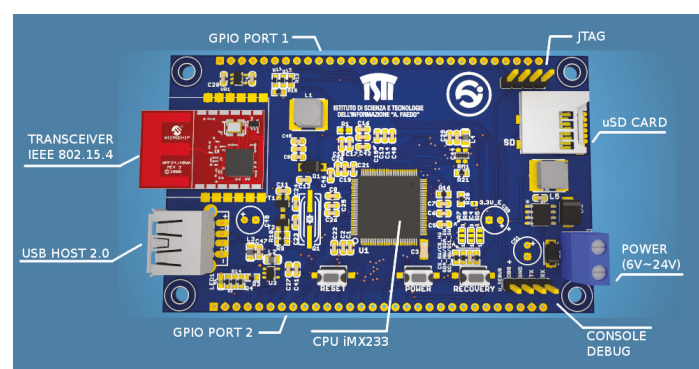

FIGURE 6: Top view design of the vision board.

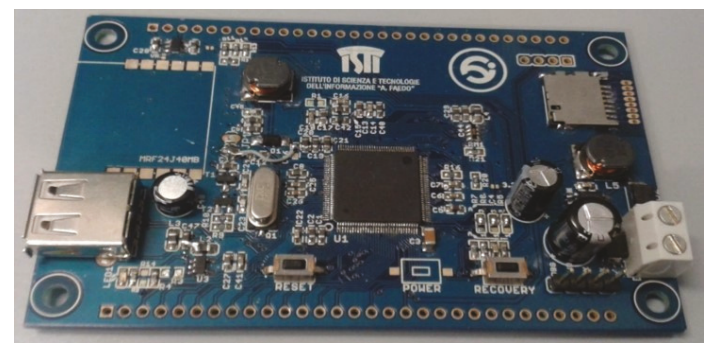

FIgURE 7: Actual vision board.

systems GNU/Linux. This architecture has the advantage to integrate within it a PMU (Power Management Unit), in addition to numerous peripheral interfaces, thus minimizing the complexity of the board. Moreover, the CPU package of type TQFP128 has helped us to minimize the layout complexity, since it was not necessary to use multilayer PCB technologies for routing. Thus, the board can be printed also in a small number of instances. The choice has contributed to the further benefit of reducing development costs; in fact, the CPU only needs an external SDRAM, a $24 \mathrm{MHz}$ quartz oscillator, and an inductance for the PMU. The chosen architecture has been proved to have an average consumption measured at the highest speed $(454 \mathrm{MHz})$ less than $500 \mathrm{~mW}$. The system includes an on-board step-down voltage regulator type LM2576 featuring high efficiency to ensure a range of voltages between 6 and $25 \mathrm{~V}$, making it ideal for batterypowered systems, in particular for power supply by lithium batteries $(7.2 \mathrm{~V}$ packs) and lead acid batteries $(6 \mathrm{~V}, 12 \mathrm{~V}$, and $24 \mathrm{~V}$ packs). A microSD slot is present, which is essential for booting the system, booting the kernel and filesystem associated (EXT4); the board can be upgraded simply by changing the contents of the microSD. On board, there are two expansion connectors (strip $1 \times 30$ ), a specific connector for the serial console of the operating system and a serial connector for debugging. On the expansion connectors, SPI, I2C, and UART peripherals are provided as well as two low resolution ADC. Figure 6 shows the top a view of the board, rendered with SketchUp directly from source Eagle CAD. The main components including the CPU iMX233, the USB host port, and the microSD card slot have been noted. Also shown are the connectors for the GPIO and debugging via serial connection. Finally, in Figure 7 the realized operational vision board is shown. 
5.2.2. The Camera. For the integration of a camera sensor on the vision board, some specific requirements were defined in the design stage for providing easiness of connection to the board itself and management through it, and capability to have at least a minimal performance in difficult visibility condition, e.g., in low light conditions. Thus the minimal constraints were as follows:

(i) An USB Video Class (UVC) device.

(ii) Possibility of removing IR filter or capability of NearIR acquisition. Moreover, the selection of a low-cost device was the main requirement considered for the whole smart camera prototype.

The USB Device Class Definition for Video Devices, or USB Video Class (USB Implementers Forum, 2012), defines video streaming functionality on the Universal Serial Bus. Similarly, to most mass storage devices (USB flash disks, external SATA disk enclosures) that can be managed by a single driver because they conform to the USB Mass Storage specification; UVC compliant peripherals only need a generic driver. Among a very large list of UVC compliant devices (see e.g. http://www.ideasonboard.org/uvc/), an easy-to-buy and cheap camera (HP HD 2300 Webcam) was selected. Such camera is equipped with an IR filter, designed to reduce the noise from IR light sources, which is easily removable for our purposes of acquiring images even in low light conditions. The camera supports several colour spaces (such as YUYV and RGB) and image resolutions up to $1280 \times 1024$. The maximum achievable frame rate is $30 \mathrm{fps}$ at $640 \mathrm{x} 480$ resolution. Through the Video For Linux 2 (V4L2) API this camera allows for several controls and commands, including brightness, contrast, saturation, hue, sharpness, and gamma correction.

5.2.3. The Wireless Communication Module. The smart camera was equipped with suitable interfaces for networking to connect to the distributed ITS architecture reported in Section 3. An approach in line with modern trends in the Internet of Things (IoT) has been followed [19] in order to be able to build a smart camera network in which each sensor provides its own interpretation of the scene. To this end, a transceiver compliant with IEEE 802.15.4 has been integrated directly on the PCB. More in detail the Microchip MRF24J40MB has been selected working in the $2.4 \mathrm{GHz}$ range and featuring data rate up to $250 \mathrm{kbps}$. Typical power consumptions of the transceiver are $25 \mathrm{~mA}$ in RX mode and $130 \mathrm{~mA}$ in TX mode. The aforementioned transceiver has an antenna integrated in the PCB; in case an external antenna is needed, the similar transceiver MRF24J40MC can be considered instead. A suitable glue has been used to integrate the transceiver with the IPv6 stack, also containing the 6LoWPAN header compression and adaptation layer for IEEE 802.15.4 links [20]. The use of IEEE 802.15.4 is an excellent solution for building a network of smart cameras in a parking lot scenario, where the nodes are not excessively far and the mesh network, also by exploiting multihop mechanisms, can guarantee a good coverage. The whole prototype with energy harvesting system is depicted in Figure 8.

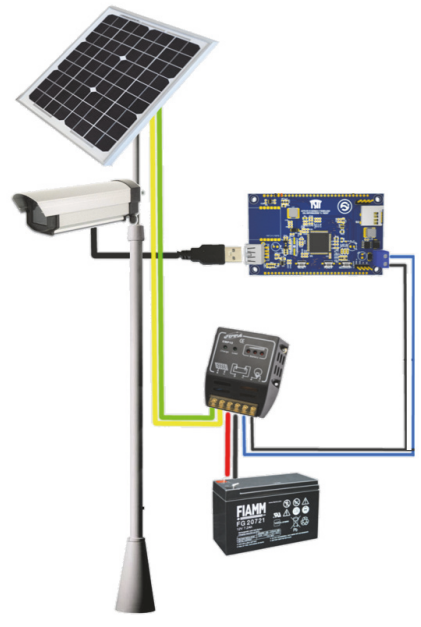

FIGURE 8: VSN sensor node prototype with energy harvesting system.

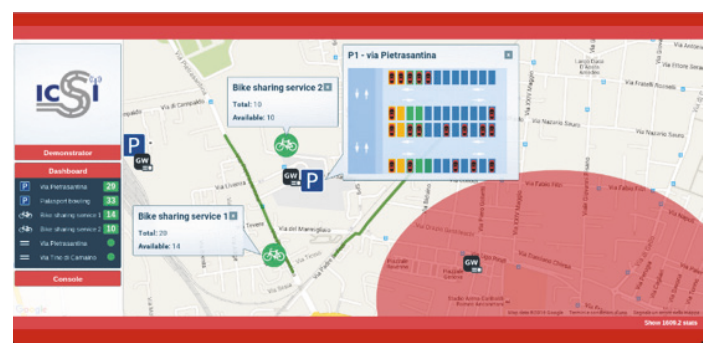

FigURE 9: Web application.

5.2.4. Energy Harvesting and Housing. The other important component of the smart camera is the power supply and energy harvesting system that controls charging and permits to choose optimal energy savings policies. The power supply system includes the lead $(\mathrm{Pb})$ acid battery pack and a module for harvesting energy through photovoltaic panel. A wide range of photovoltaic panels can be integrated, thus making possible to respond to the energy requirements in any application scenario. For the experimentation a $12 \mathrm{~V}$ panel with a nominal power of $15 \mathrm{~W}$ was used and managed by a controller outputting up to 4A DC current. The controller has protection against deep battery discharging and can deal with battery having capacity between 10 and 40Ah. A 12Ah battery has been normally used for tests.

5.3. Human-Machine Interface (HMI). A web platform and mobile applications has been developed, depicted in Figure 9, to validate the implementation and operational evaluation of the presented architecture taking into consideration integration with existing traffic management system. The main purpose of these applications is to integrate collected data from the developed wireless sensors and transmitting the aggregated results to a higher hierarchical level. A middleware has been designed and implemented over Low power Wireless Personal Area Networks (6LoWPAN) and 


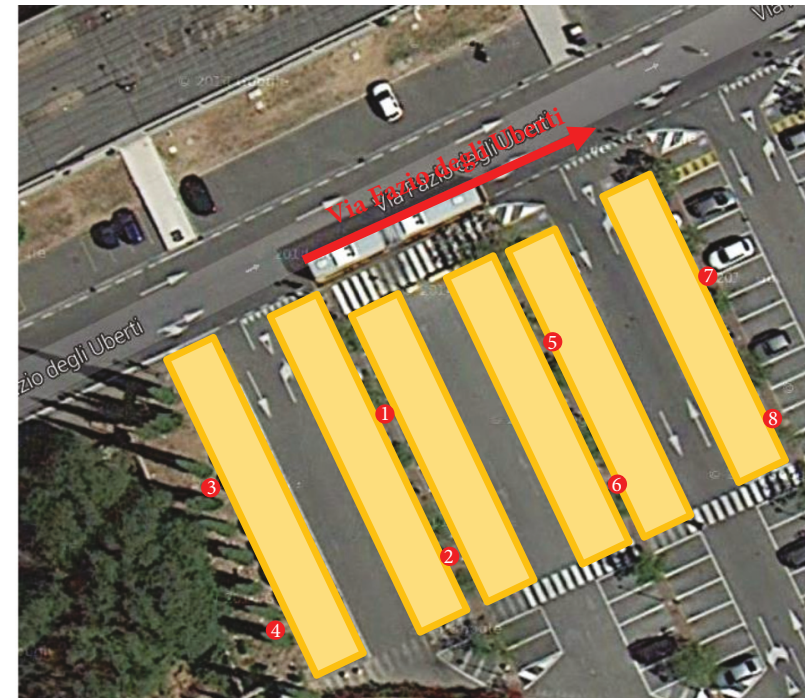

(a)

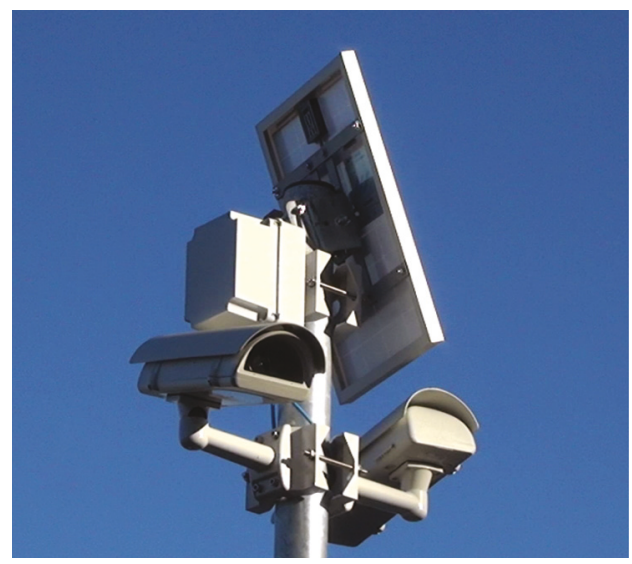

(c)

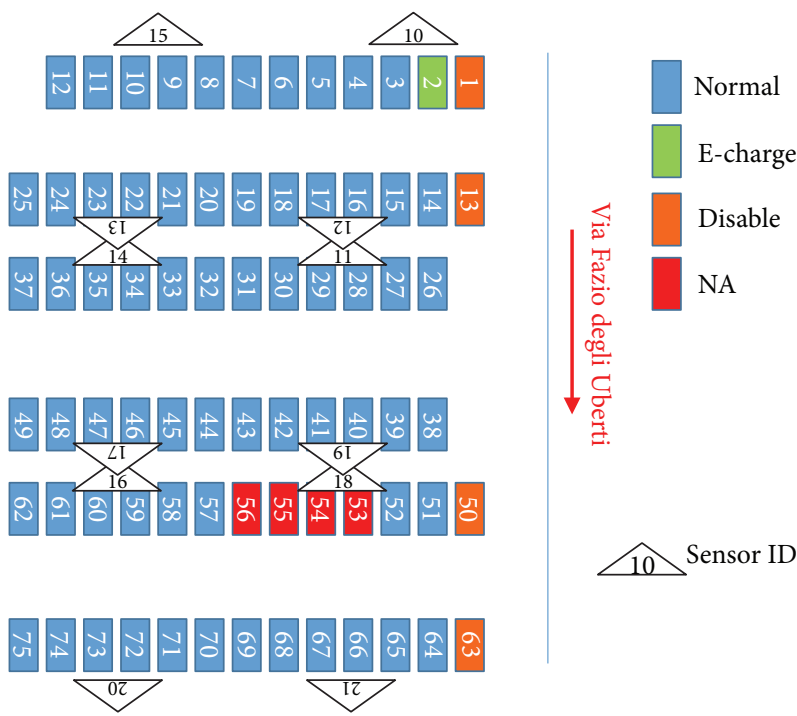

(b)

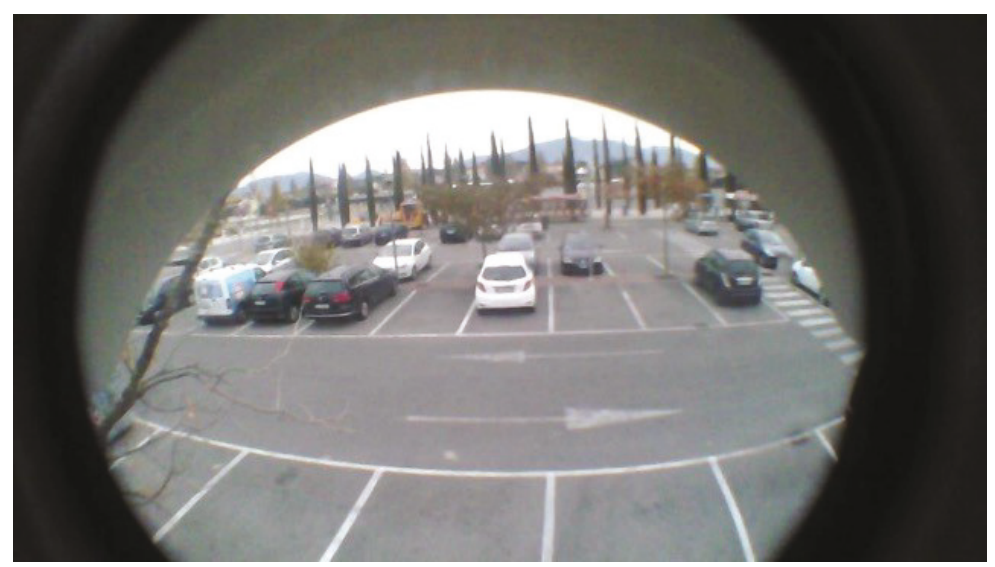

(d)

FIGURE 10: Results: (a) pole displacements for sensor installation, (b) schema of parking lot monitoring with sensor and spaces IDs, (c) example of installation of two sensors on a single pole, and (d) parking view from sensor ID = 16 .

Constrained Application Protocol (CoAP), both specialized for tiny devices, to enable web services to manipulate simple resources (e.g., vision sensors) [21]. The basic tasks that were performed are as follows: sensors monitor the status of the parking area, the GWs manage the sensed data and process it and publish the event and information messages, and the information messages are displayed via both mobile and web application.

\section{Equipment Installation}

Tests were conducted in the north-west border of the city of Pisa, Italy. The parking area is very near to Pisa main touristic attractions, including the leaning tower, to the hospital premises and to several buildings and departments of the local university. The experimental area included two subregions consisting in the main access road (on which flow analysis is performed) and a parking area subregion, on which cooperative parking monitoring is deployed. A total of 12 sensors were deployed on 8 poles as shown in Figure 10(a). These sensors monitored a total of 75 parking lots consisting of 1 Electric Cars slots, 4 Disabled slots and 70 Regular slots. The schema of these parking slots with their ids is depicted in Figure 10(b) and the deployed sensors are shown in Figure 10(c). It must be noticed that there is a number of slots which are covered by more than one sensor. On these ones, the middleware performs aggregation of the results produced by each embedded vision node according to the model described in (4).

\section{Results}

7.1. Vision Based Results. The results of the monitoring of a typical working day (November 2, 2015) scenario is shown in Figure 11(a). The monitoring, due to wintertime (i.e., short daylight time) and to the need to save the battery at nighttime, took place starting from 8:00 hours until 18:00 hours for each day. The occupancy status was recorded every 15 minutes 


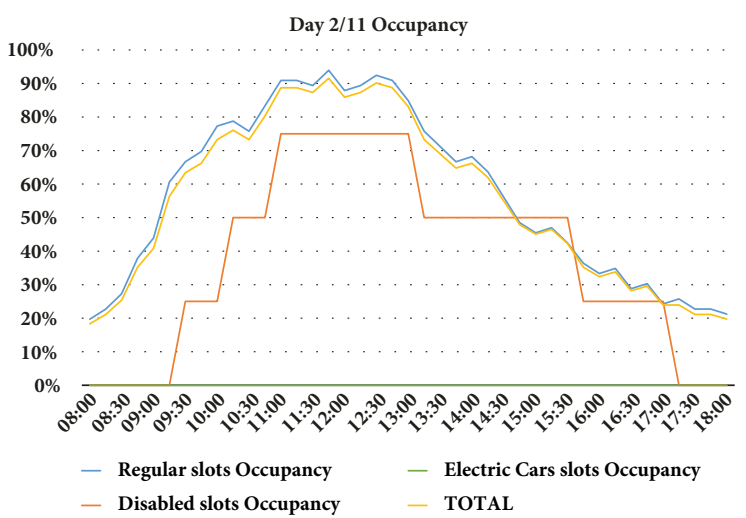

(a)

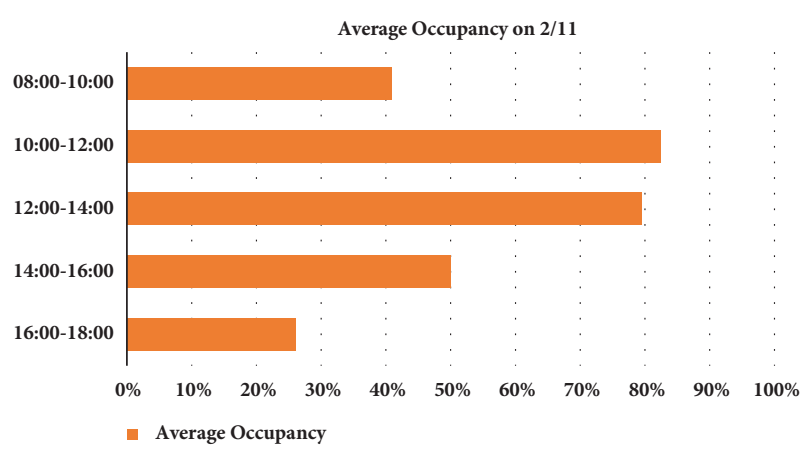

(b)

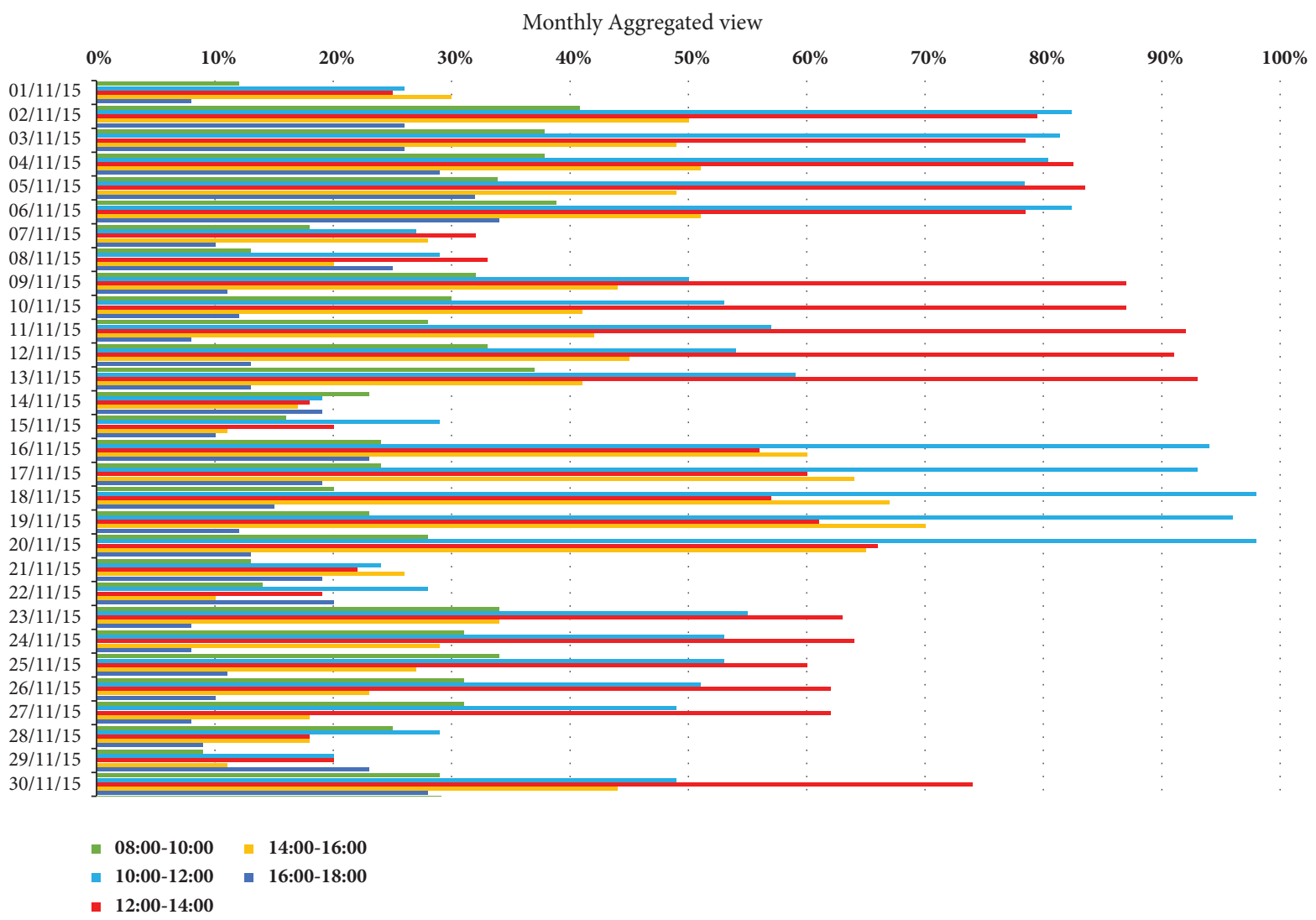

(c)

FIgURE 11: Results: (a) Occupancy status of the parking on November 2, 2015, (b) parking lot occupancy on November 2, 2015 aggregated by time slots, and (c) aggregated occupancy data by time slots for the month of November. Aggregated occupancy data by time slots for the month of December.

as depicted in Figure 11(a). The data are shown aggregated by time windows of 2 hours in Figure 11(b). The occupancy peak for that day was $92 \%$ of the capacity recorded at 11:45. As it is shown by these figures, the Electric Cars slot happened to be never occupied on that day, and this has happened several times during the monitoring; also for this reason, Pisa mobility agency had previously decided to decrease the Electric Cars available slots from two to only one.

A more extensive assessment of the results is given by a monthly view of the aggregated data and depicted in
Figure 11(c). The figure shows the whole month of November 2015. The obtained results confirm the usage of Via Pietrasantina parking as a typical long-stay swapping parking used also for mobility exchange reasons (e.g., fast bus stop link to center and train station). In fact, the occupancy ratio reaches its highest values early and quickly in the morning and only decreases slowly in the afternoon to get to its minimum towards the offices closing time. Another relevant information regards weekends low usage of the parking (i.e., never exceeding $35 \%$ of the capacity). 


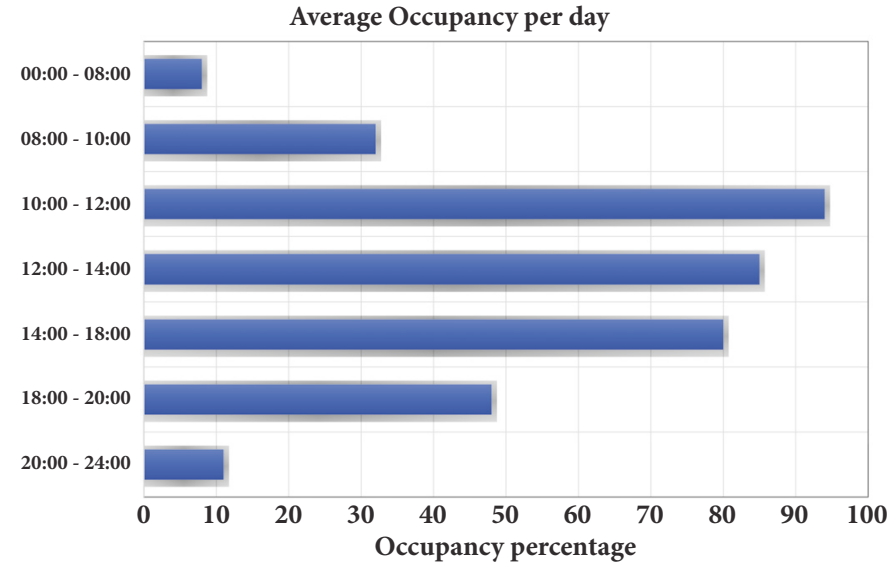

FIGURE 12: Average parking lots occupancy per day in outdoor parking.

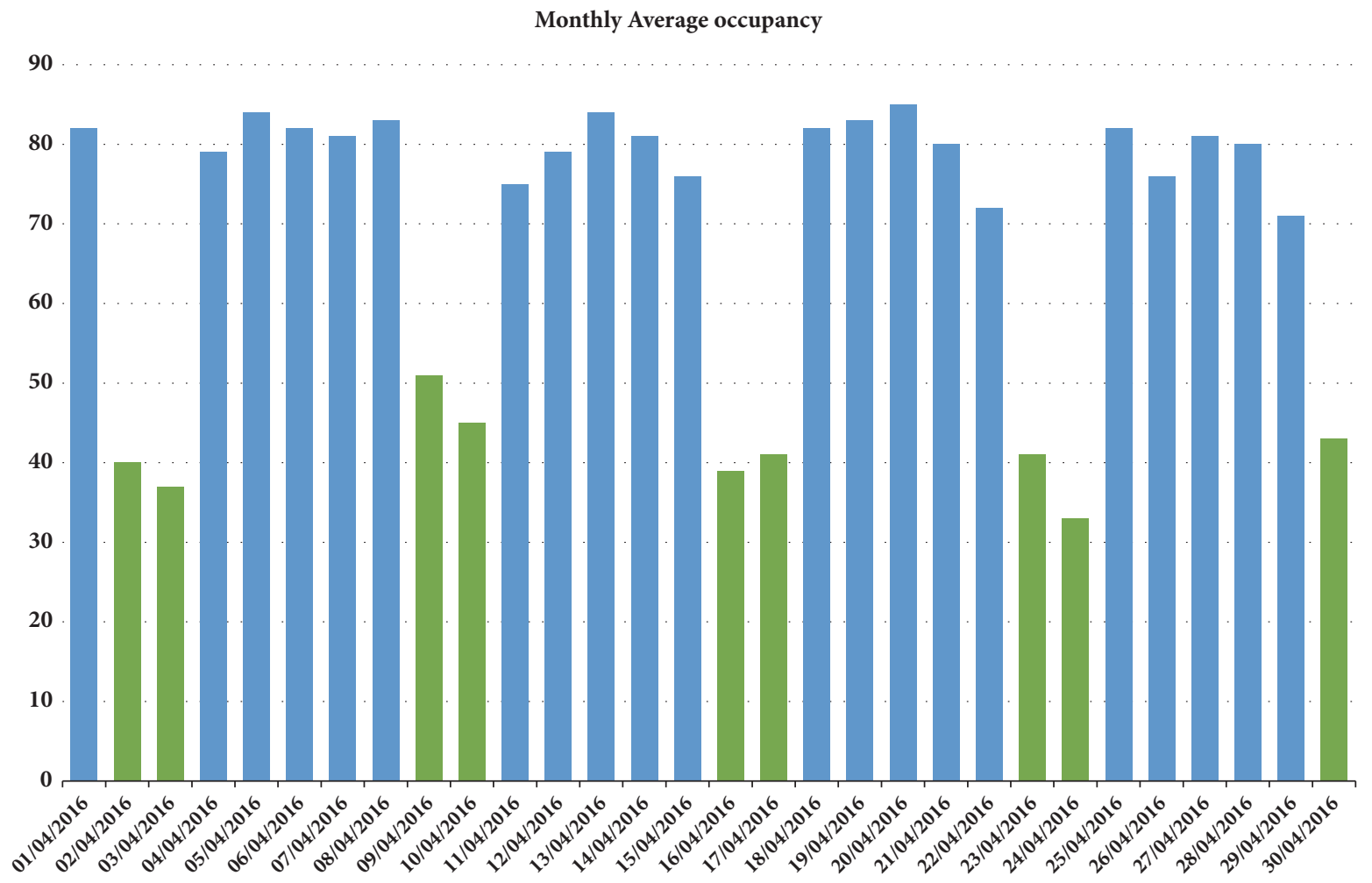

FIGURE 13: Parking occupancy for the whole month in outdoor parking.

7.2. Sensors Based Results. Sensors were deployed on an outdoor parking lot in order to avail the car parking experience on a typical working day. Figure 12 presents the average occupancy of the park on a daily basis. An overview on the graph shows a 12-hour window pattern, an higher occupancy rate between 8 and 20 followed by an abrupt decrease and almost vacancy in the remaining hours. Moreover, it can be seen that the parking lot is not used in full capacity having its maximum attendance between 10:00 and 12:00 (95\%). After this peak, the number of occupied parking slots gradually descends until reaching the end of the 12 hour window. These results and subsequent pattern can be explained by the park vicinity, mostly composed by working spaces namely: offices and the university campus. This assertion was also verified by the low car park attendance during weekends showed in the average occupancy in the month of April depicted in Figure 13. The graph also shows a slight decrease on the number of cars parked on Fridays.

A different deployment was also conducted on a private underground parking lot and integrated with the system. Six sensors were used and placed on different parking spaces, one of which was reserved for persons with disabilities (Park ID: 5048). Figure 14 shows the occupancy rate of each space per hour. The parking lot usage was very reduced throughout the 


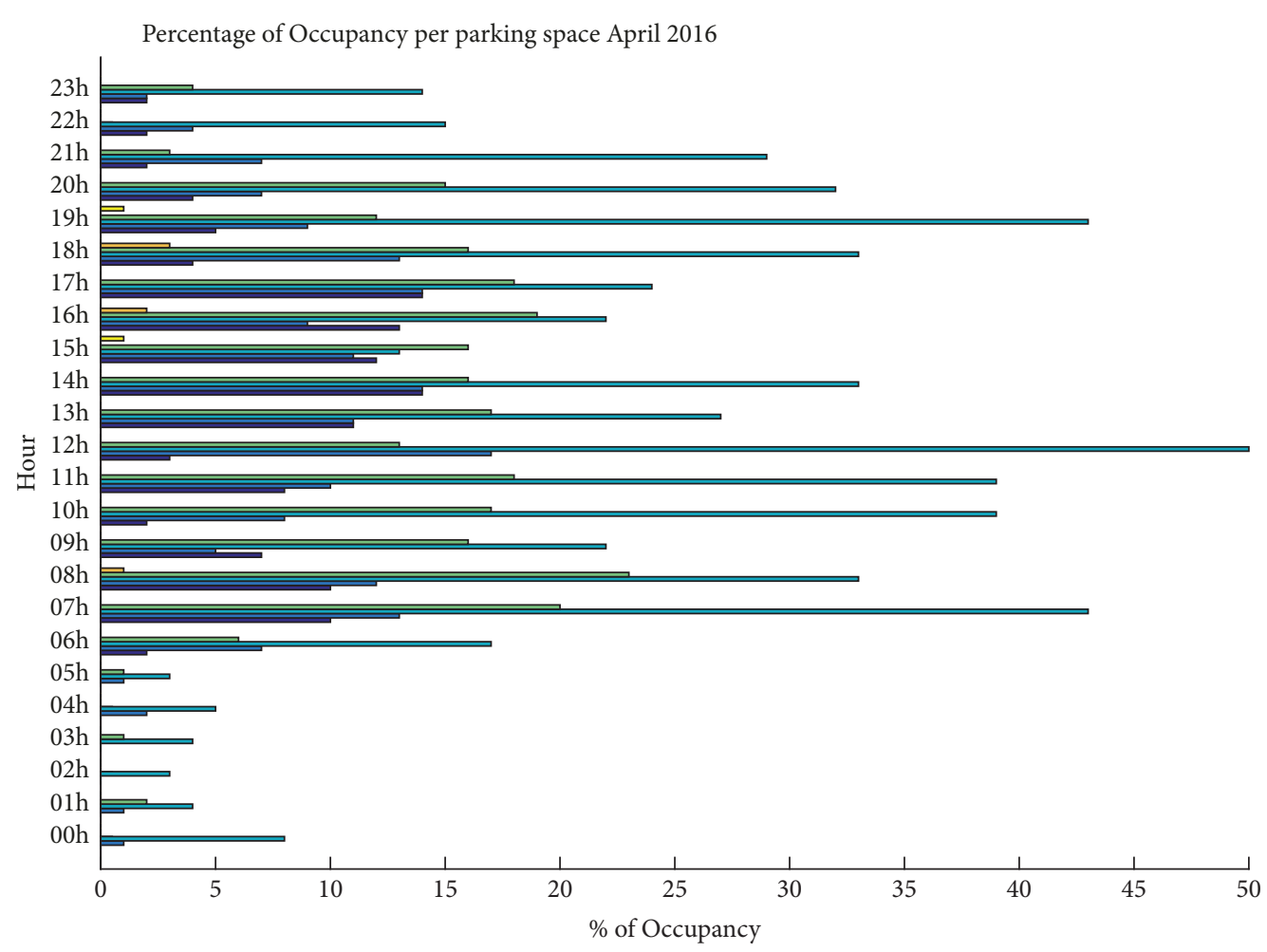

Park ID: 3

Park ID:91

Park ID:101

Park ID:102

Park ID:5058

Park ID:5048

FIGURE 14: Average parking occupancy in a day of indoor parking.

entire month, only one parking space was often used and, even so, it had a maximum occupancy of $50 \%$. Additionally, both the park spot with ID 5058 and the disabled parking space were not commonly used, specially the last one that was only occupied 11 times. These results can be justified by the lack of visibility of the car park and the lack of information regarding the number of vacant parking spaces. Thus, an application capable of providing real-time car park availability and free parking spaces for nearby drivers could improve both the parking lot usage as the entire parking experience.

\section{Conclusions}

In this paper, we have presented a fully scalable and distributed architecture that is easily integrated with multiple sensing sources.

The main novelty of the proposed approach lies in the context of scalability, and in particular the reduced costs for obtaining it and the ease of introducing a new element to the existing infrastructure. The joint design of these two aspects in our approach, in particular due to the distributed architecture designed, allows having a global complex system which, up to our knowledge, is different from the many existing and implemented in literature.

The scalability is achieved by the introduction of local gateways which have the capability of local storage. In addition, the GWs considers a hardware and software architecture fully open, modular, flexible, scalable, and capable of evolution that allows the authorities and operators to easily implement traffic management strategies and monitoring traffic based on ITS cooperative technologies.

The proposed architecture was then validated via real world deployment tests by integrating the two types of parking management systems. The magnetic sensors presented are fully encapsulated, dust/water resistant, enabling both indoor, and outdoor deployments and do not requiring any maintenance. The chosen communications technology is LoRa on the $868 \mathrm{MHz}$ band and enables low power consuming communications, but the periodicity and duration (size) of the communications was also optimized, for greater power savings. These sensors are suitable for mostly any type of car park and enable fast and simple deployments. Since they do not require infrastructure or cabling, the system becomes cheaper, involving less costs in terms of deployment 
and maintenance. The developed sensors were installed and tested in two different location: indoor parking and outdoor parking. The developed system was integrated in a novel distributed architecture in order to be able to provide scalability and an ease to the drivers in finding available parking lots, thus saving fuel and time. Scalability and reliability are granted by means of the designed distributed architecture.

Following the sensor based parking system, a smart camera prototype for vision based smart parking is also presented. Main emphasis is given to the vision board, which is a custom PCB manufactured specifically for the sensor. Cameras, wireless module, batteries, photovoltaic panels, and housing have also been described. In addition, a parking lot monitoring algorithm specifically designed for smart cameras and featuring an internode processing strategy for the final decision making is presented. 12 smart camera based sensors were deployed in on 8 poles in the city of Pisa, Italy. The parking lots were monitored for a whole month and various occupancy data were recorded: they proved to be useful both to disseminate real-time information to tourists and commuters as well to design and deploy adaptive policies for access and payment of parking lot facilities.

In the end, as already mentioned above our proposed approach seems to be quite effective in being applied to typical urban environments, in which the deployment of such infrastructures occurs gradually over time and with very different geographical dislocations.

\section{Data Availability}

Major part of the data collected and used for testing in this paper have been acquired in the course of the cited EU-FP7 Project. They can be obtained on request to the consortium composing the EU Project.

\section{Conflicts of Interest}

The authors declare that they have no conflicts of interest.

\section{Acknowledgments}

The research leading to these results has received funding from the European Union's Seventh Framework Programme (FP7) under Grant Agreement no. 3176711.

\section{References}

[1] EU, Passenger cars in the EU - Statistics Explained - European Commission, http://ec.europa.eu/eurostat/, Accessed on: 15 May 2017.

[2] US. Data and Statistics - Bureau of Transportation Statistics, URL: https://www.rita.dot.gov/bts/data_and_statistics/index .html, Accessed on: 15 May 2017.

[3] M. Magrini, D. Moroni, G. Pieri, and O. Salvetti, "Smart cameras for ITS in urban environment," Intelligent Transport Systems: Technologies and Applications, pp. 167-188, 2015.

[4] SIEMENS, Future of Infrastructure: The Smart Way to Park, last visited: June 2016.

[5] M. Kodransky and G. Hermann, Europe's Parking U-Turn: From Accommodation to Regulation, Institute for Transportation and
Development Policy, 2011, https://www.itdp.org/wp-content/ uploads/2014/07/Europes_Parking_U-Turn_ITDP.pdf, Accessed on: 15 September, 2017.

[6] V. W. S. Tang, Y. Zheng, and J. Cao, "An intelligent car park management system based on wireless sensor networks," in Proceedings of the SPCA 2006: 1st International Symposium on Pervasive Computing and Applications, pp. 65-70, August 2006.

[7] T. Lin, H. Rivano, and F. Le Mouel, "A survey of smart parking solutions," IEEE Transactions on Intelligent Transportation Systems, vol. 18, no. 12, pp. 3229-3253, 2017.

[8] L. Ruizhi, R. Cristian, B. Peter, O. Shumao, and C. Liping, "Crowdsourcing on-street parking space detection," https:// arxiv.org/abs/1603.00441, 2016.

[9] J. Vera-Gómez, A. Quesada-Arencibia, C. García, R. Suárez Moreno, and F. Guerra Hernández, "An Intelligent Parking Management System for Urban Areas," Sensors, vol. 16, no. 6, p. 931, 2016.

[10] G. Amato, F. Carrara, F. Falchi, C. Gennaro, and C. Vairo, "Car parking occupancy detection using smart camera networks and Deep Learning," in Proceedings of the 2016 IEEE Symposium on Computers and Communication, ISCC 2016, pp. 1212-1217, July 2016.

[11] E. C. Neto, E. S. Reboucas, J. L. De Moraes, S. L. Gomes, and P. P. R. Filho, "Development control parking access using techniques digital image processing and applied computational intelligence," IEEE Latin America Transactions, vol. 13, no. 1, pp. 272-276, 2015.

[12] G. Maria, E. Baccaglini, D. Brevi, M. Gavelli, and R. Scopigno, "A drone-based image processing system for car detection in a smart transport infrastructure," in Proceedings of the 18th Mediterranean Electrotechnical Conference, MELECON 2016, pp. 1-5, April 2016.

[13] Q. Wu, C. Huang, S.-Y. Wang, W.-C. Chiu, and T. Chen, "Robust parking space detection considering inter-space correlation," in Proceedings of the IEEE International Conference on Multimedia and Expo, ICME 2007, pp. 659-662, July 2007.

[14] S. Funck, N. Möhler, and W. Oertel, "Determining car-park occupancy from single images," in Proceedings of the 2004 IEEE Intelligent Vehicles Symposium, pp. 325-328, June 2004.

[15] C.-C. Huang, Y.-S. Dai, and S.-J. Wang, "A surface-based vacant space detection for an intelligent parking lot," in Proceedings of the 2012 12th International Conference on ITS Telecommunications, ITST 2012, pp. 284-288, IEEE, November 2012.

[16] C. Gálvez del Postigo, J. Torres, and J. M. Menéndez, "Vacant parking area estimation through background subtraction and transience map analysis," IET Intelligent Transport Systems, vol. 9, no. 9, pp. 835-841, 2015.

[17] Intelligent Cooperative Sensing for Improved traffic efficiency, http://www.ict-icsi.eu/.

[18] M. Magrini, D. Moroni, G. Palazzese, G. Pieri, G. Leone, and O. Salvetti, "Computer Vision on Embedded Sensors for Traffic Flow Monitoring," in Proceedings of the 18th IEEE International Conference on Intelligent Transportation Systems, ITSC 2015, pp. 161-166, September 2015.

[19] Z. Shelby, "Embedded web services," IEEE Wireless Communications Magazine, vol. 17, no. 6, pp. 52-57, 2010.

[20] Z. Shelby and C. Bormann, 6LoWPAN: The wireless embedded Internet, vol. 43, John Wiley \& Sons, 2011.

[21] A. Azzara, M. Petracca, and P. Pagano, "The icsi m2m middleware for iot-based intelligent transportation systems," in Proceedings of the 2015 IEEE 18th International Conference on Intelligent Transportation Systems, pp. 155-160, 2015. 


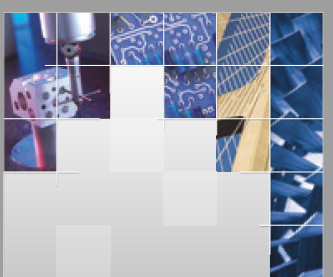

\section{Enfincering}
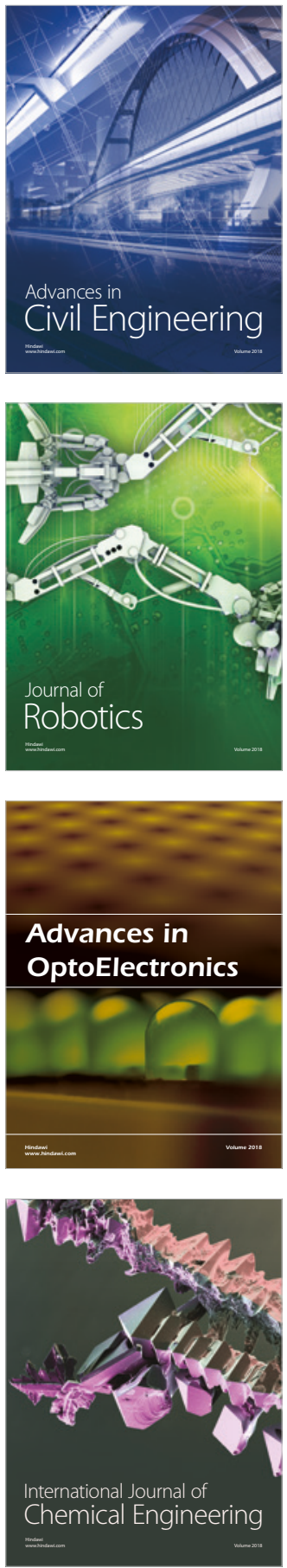

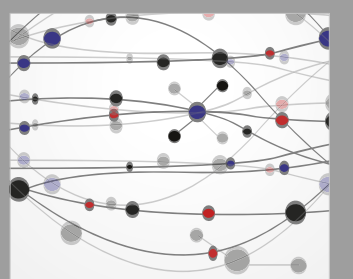

\section{Rotating \\ Machinery}

The Scientific World Journal

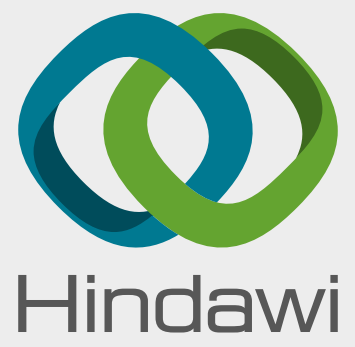

Submit your manuscripts at

www.hindawi.com
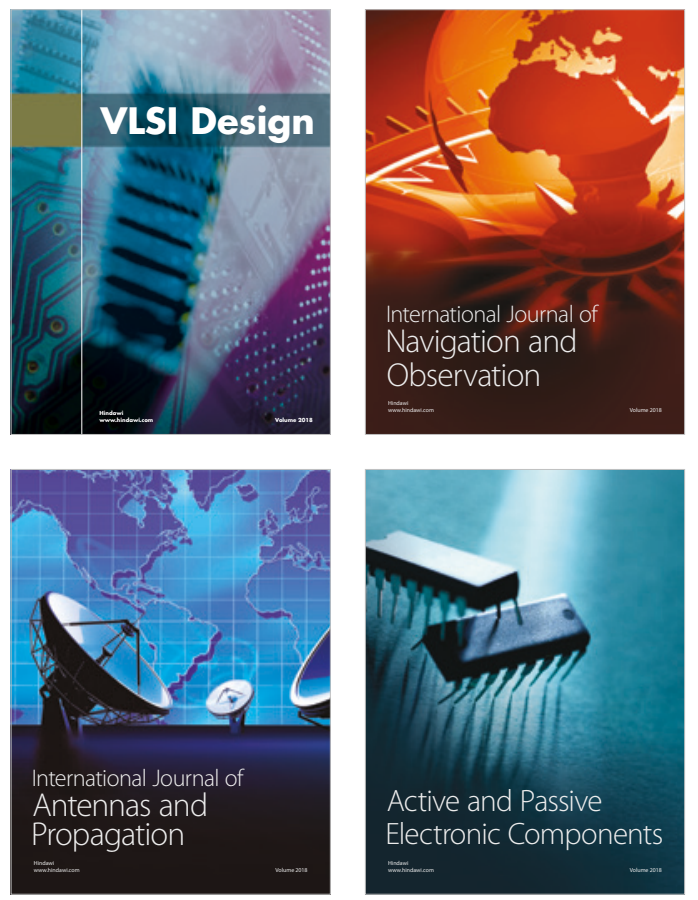
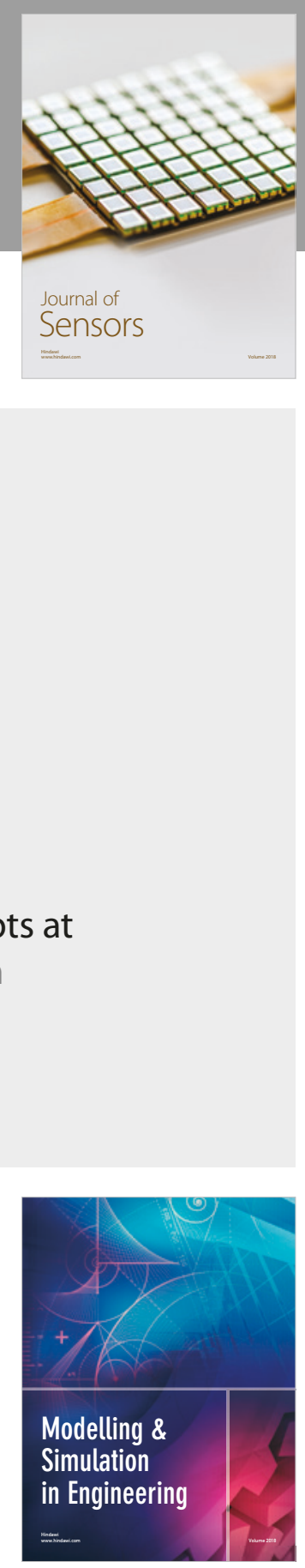

\section{Advances \\ Multimedia}
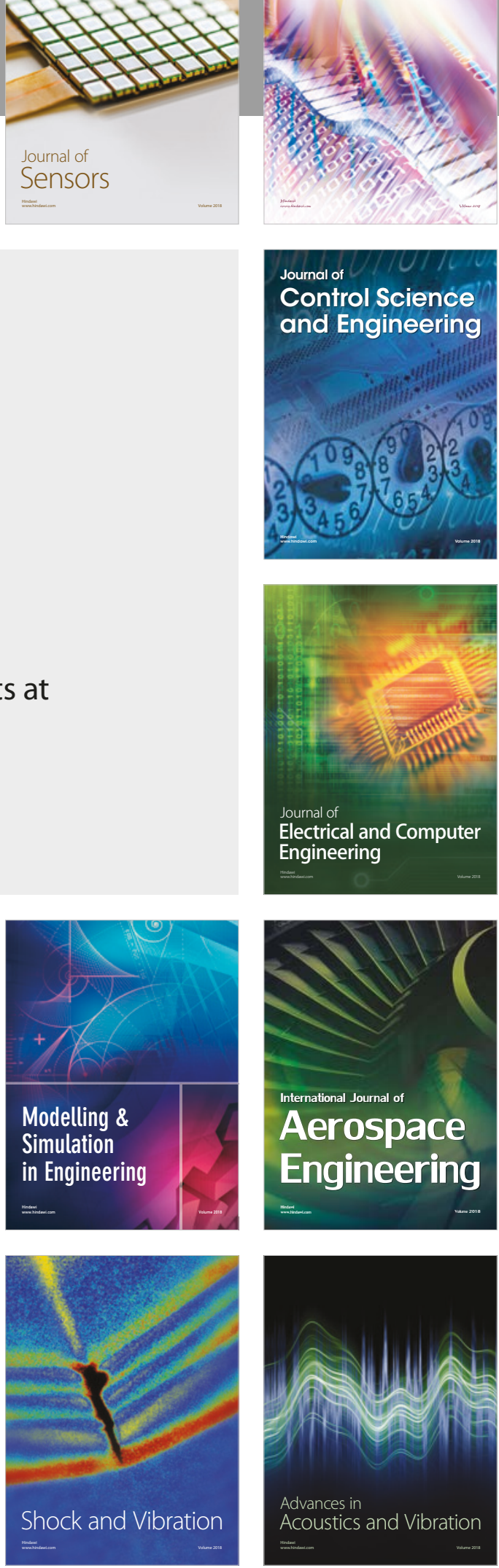